\title{
Prevotella oralis
}

National Cancer Institute

\section{Source}

National Cancer Institute. Prevotella oralis. NCI Thesaurus. Code C86675.

A species of obligately anaerobic, Gram-negative, rod shaped bacteria assigned to the phylum Bacteroidetes. This species is nonmotile, non-spore forming, nonpigmented, cellobiose, rhamnose, salicin sucrose, lactose, esculin hydrolysis, beta- $\mathrm{N}$-acetylglucosaminidase, alpha-fucosidase, beta-glucosidase, and glycine aminopeptidase positive, but xylose, arabinose, indole, and beta-xylosidase negative. P. oralis is part of the normal human oral cavity flora, but is associated with oral and upper respiratory infections. 